\title{
Haemodynamic effects of nasal continuous positive airway pressure in severe congestive heart failure
}

\author{
R. Liston*, P.C. Deegan*, C. McCreery ${ }^{\dagger}$, R. Costello*, B. Maurer ${ }^{\dagger}$, W.T. McNicholas*
}

Haemodynamic effects of nasal continuous positive airway pressure in severe congestive heart failure. R. Liston, P.C. Deegan, C. McCreery, R. Costello, B. Maurer, W.T. McNicholas. CERS Journals Ltd 1995.

ABSTRACT: Nasal continuous positive airway pressure (NCPAP) during sleep may be a useful adjunct to medical therapy in patients with stable severe congestive heart failure (CHF), particularly when there is a coexisting respiratory sleep disorder. However, the direct haemodynamic effects of NCPAP in patients with severe stable CHF have not yet been adequately assessed.

Right heart catheter studies were performed in seven awake males (aged 51-75 yrs) with stable CHF, before, during and after the application of $5 \mathrm{cmH}_{2} \mathrm{O}$ NCPAP over $3 \mathrm{~h}$. All patients had left ventricular ejection fractions $\leq 30 \%$ and baseline pulmonary capillary wedge pressures $>12 \mathrm{mmHg}$, and six patients were in atrial fibrillation.

Cardiac index fell from baseline in all patients whilst on NCPAP, with the greatest fall at $2 \mathrm{~h}$ (from $3.3 \pm 0.3$ (mean \pm SEM) at baseline to $2.8 \pm 0.2 \mathrm{l} \cdot \mathrm{min}^{-1} \cdot \mathrm{m}^{-2}$ ) and rose back to baseline after NCPAP withdrawal. Systemic vascular resistance (SVR) increased during NCPAP application $\left(1,268 \pm 108\right.$ to $\left.1,560 \pm 82 \mathrm{dyn} \cdot \mathrm{s}^{-1} \cdot \mathrm{cm}^{5}\right)$, with baseline SVR showing a significant negative correlation $v s$ percentage fall in cardiac index $(\mathrm{CI})$ at $2 \mathrm{~h}$ on multiple linear regression analysis $\left(\mathrm{r}^{2}=0.8\right)$.

These data indicate that domiciliary nocturnal NCPAP should not be prescribed as part of the therapy in severe $\mathrm{CHF}$ without first determining the individual patient's cardiac response to such therapy.

Eur Respir J., 1995, 8, 430-435.

Nasal continuous positive airway pressure (NCPAP) is an effective therapy for obstructive sleep apnoea [1, 2] and some forms of central sleep apnoea [3]. Positive airway pressure has also been reported to reduce left ventricular transmural pressure in the failing heart, with beneficial effects on cardiac output [4-6]. These findings suggest that NCPAP may be beneficial in the treatment of congestive heart failure (CHF), and indeed several previous reports have documented benefits from various forms of positive airway pressure administered acutely in some patients with acute pulmonary oedema [7-10], or prior to cardiac transplantation in patients with severe chronic CHF [11]. In addition, two short-term studies of domiciliary nocturnal NCPAP have demonstrated beneficial effects on cardiac function in patients with CHF and sleep-disordered breathing $[12,13]$, although another recent study has failed to demonstrate benefit from this therapy [14].

The above reports raise the possibility that domiciliary nocturnal NCPAP may be a useful adjunctive therapy in patients with chronic severe CHF. The mechanism of such benefit could be either direct, through a beneficial effect of NCPAP on cardiac function, or indirect, through a beneficial cardiac effect of the relief of hypoxaemia and/or sleep-disordered breathing, particularly among patients with sleep apnoea or Cheyne-Stokes res-
Depts of *Respiratory Medicine and Cardiology, University College and St. Vincent's Hospital, Dublin, Ireland.

Correspondence: W.T. McNicholas Dept of Respiratory Medicine St. Vincent's Hospital

Elm Park

Dublin 4

Ireland

Keywords: Cardiac output

heart failure

nasal continuous positive airway pressure

Received: July 141994

Accepted after revision December 11994 piration. Support for a direct beneficial effect of NCPAP on cardiac function comes from the findings of BRADLEY et al. [15]. They found that the application of NCPAP for $10 \mathrm{~min}$ in awake patients with stable $\mathrm{CHF}$ and in sinus rhythm, was associated with a significant increase in cardiac index (CI), but only in a subgroup of patients with high pulmonary capillary wedge pressures. However, if NCPAP is to be used overnight in the clinical setting among patients with severe CHF, the haemodynamic effects of NCPAP need to be assessed over a longer time period than $10 \mathrm{~min}$. In addition, haemodynamic effects may vary at different pressure levels, and may differ among patients with CHF who are not in sinus rhythm.

We therefore studied the time course of effects of NCPAP on haemodynamic variables, both during and after its application over a longer time period $(3 \mathrm{~h})$ in patients with severe but stable CHF. These patients were on maximum medical therapy, but still demonstrated severe exercise impairment.

\section{Patients and methods}

\section{Patients}

Consecutive patients admitted to the cardiology department for assessment of $\mathrm{CHF}$ were considered for 
enrolment. Entry criteria included a clinical diagnosis of stable CHF, class 3 or 4 cardiac dyspnoea by the New York Heart Association Classification [16], and a left ventricular ejection fraction (LVEF) of $30 \%$ or less by echocardiography. Exclusion criteria included significant primary lung disease or unstable cardiac status, in addition to CHF secondary to valvular heart disease, or specific nasal problems that prevented the comfortable use of NCPAP. All patients gave written informed consent, and the study was approved by the Hospital Ethics Committee.

Eight patients were enrolled, and all were current nonsmokers. Cardioactive medication was unaltered in the 3 day period prior to haemodynamic studies, and none had evidence of acute cardiac ischaemia or respiratory failure. Seven patients were in atrial fibrillation (AF), and one was in sinus rhythm (SR). All had full spirometry using a computerized system (TT Autolink, PK Morgan, Gillingham, UK). Six of the seven patients had full overnight sleep studies performed, using standard polysomnographic techniques $[17,18]$ to assess the presence or absence of any associated respiratory sleep disorder, such as sleep apnoea or Cheyne-Stokes respiration. Patients were familiarized with the NCPAP apparatus on the day prior to the study, and arterial blood gases were taken in the recumbent position immediately prior to insertion of the cardiac catheter.

\section{Haemodynamic studies}

The haemodynamic studies were performed in a quiet private room in the Coronary Care Unit with the patient awake and supine, and the last dose of diuretic given at least $4 \mathrm{~h}$ previously. A 7 Fr flow-directed thermodilution catheter (Multiflex TD thermodilution catheter; Abbott Critical Care Systems, Nth Chicago, Il, USA) was inserted via the left subclavian venous route. Before administration of NCPAP, baseline readings (time zero) were taken a minimum of $15 \mathrm{~min}$ after insertion of the catheter and after heart rate (HR) and blood pressure (BP) had returned to baseline levels. These readings included right atrial pressure (RAP), pulmonary artery pressures (PAPs), and pulmonary capillary wedge pressure (PCWP) recorded at end-expiration, in addition to $\mathrm{HR}$ and $\mathrm{BP}$. Cardiac output (CO), cardiac index (CI), stroke volume (SV) and stroke volume index (SVI) were measured by the thermodilution technique [19], using normal saline cooled to $0^{\circ} \mathrm{C}$, and taking the mean of five readings. Systemic vascular resistance (SVR) and pulmonary vascular resistance (PVR) were then calculated using standard formulae [20]. Nasal CPAP (REAlstar ${ }^{\mathrm{TM}}$; Respironics Inc., Murrayville, PA, USA) was then applied, using a comfortably fitting nasal mask at an initial pressure of 5 $\mathrm{cmH}_{2} \mathrm{O}[10,15]$. Patients were encouraged to keep their mouth closed, in order to ensure full transmission of mask pressure to the lungs [21], and to remain awake.

The above measurements were repeated after $0.25,1$, 1.5 and $2 \mathrm{~h}$ of NCPAP. At this point, the protocol dictated an increase in the NCPAP pressure level to 10 $\mathrm{cmH}_{2} \mathrm{O}$ among patients who showed either no change or an increase in $\mathrm{CI}$ during $5 \mathrm{cmH}_{2} \mathrm{O}$ NCPAP, and readings were repeated at the same time intervals as for the $5 \mathrm{cmH}_{2} \mathrm{O}$ pressure level. Patients who demonstrated a decrease in CI during $5 \mathrm{cmH}_{2} \mathrm{O}$ NCPAP were to be continued at this pressure level for a further $1 \mathrm{~h}$. The NCPAP was then discontinued, patients remained supine and readings were taken after a further 0.25 and 0.75 h.

Arterial oxygen saturation $\left(\% \mathrm{SaO}_{2}\right)$ was monitored throughout the study period by pulse oximetry (Biox 3700; Ohmeda, Louisville, CO, USA).

\section{Statistical analysis}

The differences between the means of haemodynamic variables at each recording point during the study period were analysed using analysis of variance (ANOVA) for repeated measures. Post hoc analysis was performed using the Student Newman-Keuls (SNK) test for multiple comparisons to detect significant differences between baseline and each time-point. In order to determine which independent variables measured at baseline were correlated with the percentage fall in cardiac index (CI) whilst on NCPAP, stepwise multiple linear regression analysis was performed for CI at 0.25 and $2 \mathrm{~h}$, and at the end of NCPAP application. Independent variables entered into the regression included age, LVEF, forced expiratory volume in one second/forced vital capacity $\left(\mathrm{FEV}_{1} / \mathrm{FVC}\right)$ ( $\%$ predicted), baseline arterial oxygen tension $\left(\mathrm{PaO}_{2}\right)$, RAP, PCWP, CI, SVI, SVR and PVR. These analyses were performed using the software package Statistica for Windows Release 4 (Statsoft, Inc. 1993). A significant value was taken as $\mathrm{p}<0.05$. Data are expressed as mean \pm SEM.

\section{Results}

Seven patients completed the study protocol, and their anthropometric and baseline pulmonary function data are outlined in table 1 . The eighth subject (in AF) withdrew during the course of haemodynamic studies due to discomfort. There had been considerable technical difficulties with the insertion of the right heart catheter in this patient, which had significantly delayed the application of NCPAP. The patient found the prolonged period of confinement in the one position uncomfortable, and asked to discontinue the protocol because of this discomfort. His data were excluded from the final analysis.

All of the remaining seven patients were male, and none was significantly hypoxaemic at rest. On spirometry, two patients had mild airflow obstruction, and a third had a restrictive deficit. None of these patients had evidence of a primary respiratory disorder or were on respiratory medication, and it was felt that the abnormal spirometry was a consequence of CHF. Table 2 summarizes the patients' cardiovascular status. All were judged by their attending cardiologist to be on maximum medical therapy at the time of study. Whilst three 
Table 1. - Anthropometric, spirometric, arterial blood gas and sleep data

\begin{tabular}{|c|c|c|c|c|c|c|c|c|c|c|c|}
\hline $\begin{array}{l}\text { Subject } \\
\text { No. }\end{array}$ & $\begin{array}{l}\text { Age } \\
\text { yrs }\end{array}$ & Sex & $\begin{array}{c}\text { Weight } \\
\text { kgs }\end{array}$ & $\begin{array}{c}\text { Height } \\
\mathrm{m}\end{array}$ & $\begin{array}{c}\mathrm{BMI} \\
\mathrm{kg} \cdot \mathrm{m}^{-2}\end{array}$ & $\begin{array}{l}\mathrm{FEV}_{1} \\
\% \text { pred }\end{array}$ & $\begin{array}{l}\mathrm{FVC} \\
\% \text { pred }\end{array}$ & $\begin{array}{c}\mathrm{FEV}_{1} / \mathrm{FVC} \\
\% \text { pred }\end{array}$ & $\begin{array}{l}\mathrm{PaO}_{2} \\
\mathrm{kPa}\end{array}$ & $\begin{array}{c}\mathrm{PaCO}_{2} \\
\mathrm{kPa}\end{array}$ & AHI \\
\hline 1 & 64 & M & 60 & 1.60 & 23.4 & 41 & 49 & 84 & 14.2 & 3.51 & $1^{*}$ \\
\hline 2 & 69 & M & 98 & 1.82 & 29.6 & 101 & 96 & 105 & 12.0 & 4.34 & $16^{\dagger}$ \\
\hline 3 & 51 & M & 63 & 1.73 & 21.0 & 59 & 91 & 65 & 11.4 & 5.53 & $3^{*}$ \\
\hline 4 & 52 & M & 79 & 1.73 & 26.4 & 113 & 106 & 110 & 13.3 & 4.96 & $22^{\dagger}$ \\
\hline 5 & 75 & $\mathrm{M}$ & 78 & 1.80 & 24.1 & 73 & 106 & 70 & 10.7 & 5.05 & $5^{*}$ \\
\hline 6 & 57 & M & 70 & 1.66 & 25.4 & 89 & 93 & 99 & 12.6 & 4.99 & $26^{\dagger}$ \\
\hline 7 & 57 & $\mathrm{M}$ & 65 & 1.65 & 23.8 & 56 & 65 & 91 & 12.5 & 4.56 & - \\
\hline
\end{tabular}

$\mathrm{M}$ : male; $\mathrm{FEV}_{1}$ : forced expiratory volume in one second; $\mathrm{FVC}$ : forced vital capacity $\mathrm{PaO}_{2}$ : arterial oxygen tension; $\mathrm{PaCO}$ : arterial carbon dioxide tension. BMI: body mass index (weight $/$ height $^{2}$ ); $\%$ pred: percentage predicted; AHI: apnoea/hypopnoea frequency per hour of sleep $\left(*\right.$ : normal $<15$ events $\cdot \mathrm{h}^{-1} ;{ }^{\dagger}$ : mild sleep apnoea $15-30$ events $\left.\cdot \mathrm{h}^{-1}\right)$.

Table 2. - Baseline cardiac function and medications

\begin{tabular}{lcccl}
\hline $\begin{array}{l}\text { Subject } \\
\text { No. }\end{array}$ & $\begin{array}{c}\text { Aetiology } \\
\text { of CHF }\end{array}$ & LVEF & $\begin{array}{c}\text { NYHA } \\
\text { Grade }\end{array}$ & Medications \\
\hline 1 & ICM & $15 \%$ & 4 & LD, Ami \\
2 & IHD & $30 \%$ & 3 & LD, Dig, AI \\
3 & ICM & $18 \%$ & 3 & LD, Dig, AI \\
4 & ICM & $25 \%$ & 3 & LD, Dig, AI \\
5 & ICM & $28 \%$ & 3 & LD, Dig \\
6 & ICM & $25 \%$ & 3 & LD, Dig, AI \\
7 & IHD & $22 \%$ & 3 & LD, Dig, AI \\
\hline
\end{tabular}

CHF: congestive heart failure; ICM: idiopathic cardiomyopathy; IHD: ischaemic heart disease; LVEF: left ventricular ejection fraction; NYHA: New York Heart Association Classification; LD: loop diuretic; Ami: amiodarone; Dig: digoxin; AI: angiotensin converting enzyme (ACE) inhibitor.

patients (table 1) had an apnoea and hypopnoea frequency within the range of mild sleep apnoea as conventionally defined [18], none had significant oxygen desaturation during sleep, and none had symptoms suggestive of a sleep apnoea syndrome. No patient had evidence of Cheyne-Stokes respiration.

\section{Haemodynamic data}

The effects of NCPAP on haemodynamic variables are outlined in table 3 . As CI fell in all patients during the initial $2 \mathrm{~h}$ period, NCPAP was not increased to 10 $\mathrm{cmH}_{2} \mathrm{O}$ in any patient, and further measurements were made at 2.5 and $3 \mathrm{hr}$ on $5 \mathrm{cmH}_{2} \mathrm{O}$ before discontinuing NCPAP. Cardiac index fell in all patients whilst on NCPAP ( $\mathrm{p}=0.0007$ by ANOVA), and returned towards baseline values after discontinuation. Individual patient data for CI during and after NCPAP are illustrated in figure 1. Multiple comparisons testing showed a significant decrease in $\mathrm{CI}$ at each recording point whilst on NCPAP, which was maximum at $2 \mathrm{~h}(\mathrm{p}<0.01)$. Cardiac index at $3.75 \mathrm{~h}$ (45 min after cessation of NCPAP) showed no significant difference from baseline, indicating that it was the NCPAP that was responsible for the observed changes, and not the supine body position.

One patient, who was in SR, did show a small improvement at $3 \mathrm{~h}\left(\mathrm{CI}=2.6 \mathrm{l} \cdot \mathrm{min}^{-1} \cdot \mathrm{m}^{-2}\right)$ compared to baseline (2.4). However, he experienced a fall at $0.25 \mathrm{~h}$ to $2.0 l \cdot \mathrm{min}^{-1} \cdot \mathrm{m}^{-2}$, and the subsequent gradual increase in $\mathrm{CI}$ over the next $2.75 \mathrm{~h}$ was due almost exclusively

Table 3. - Haemodynamic data before, during and after NCPAP, $5 \mathrm{cmH}_{2} \mathrm{O}$

\begin{tabular}{|c|c|c|c|c|c|c|c|c|c|c|c|c|c|}
\hline \multirow[b]{2}{*}{ CI } & \multirow[b]{2}{*}{$l \cdot \mathrm{min}^{-1} \cdot \mathrm{m}^{-2}$} & \multicolumn{2}{|c|}{ Baseline } & \multicolumn{2}{|c|}{$15 \mathrm{~min}$} & \multicolumn{2}{|c|}{$1 \mathrm{~h}$} & \multicolumn{2}{|c|}{$2 \mathrm{~h}$} & \multicolumn{2}{|c|}{$3 \mathrm{~h}$} & Off NCPAP & \multirow{2}{*}{$\begin{array}{c}\text { ANOVA } \\
0.0007\end{array}$} \\
\hline & & 3.3 & $(0.3)$ & 2.9 & $(0.3)^{*}$ & 2.8 & $(0.2)^{* *}$ & 2.8 & $(0.2)^{* *}$ & 2.9 & $(0.2)^{*}$ & $(0.3)$ & \\
\hline SVI & $\mathrm{ml} \cdot \mathrm{m}^{-2}$ & & (4) & 39 & (4) & 35 & $(3)^{*}$ & 35 & $(3)^{*}$ & 37 & (4) & $38 \quad$ (4) & 0.03 \\
\hline SBP & $\mathrm{mmHg}$ & 131 & (7) & 137 & (9) & 141 & $(10)^{*}$ & 149 & $(10)^{* *}$ & 149 & $(10)^{* *}$ & $144(10)^{*}$ & 0.0006 \\
\hline DBP & $\mathrm{mmHg}$ & 88 & (4) & 89 & (3) & 91 & (4) & 91 & (4) & 91 & (6) & $90 \quad$ (4) & NS \\
\hline HR & beats $\cdot \min ^{-1}$ & & (6) & 80 & (7) & 82 & (6) & 85 & (6) & 86 & (8) & (6) & NS \\
\hline SVR & $\mathrm{dyn} \cdot \mathrm{s}^{-1} \cdot \mathrm{cm}^{5}$ & 1268 & (108) & 1480 & $(160)^{\dagger}$ & 1538 & $(155)^{* *}$ & 1560 & $(82)^{* *}$ & 1501 & $(119)^{*}$ & 1368 (142) & 0.003 \\
\hline PVR & $\mathrm{dyn} \cdot \mathrm{s}^{-1} \cdot \mathrm{cm}^{5}$ & 135 & (32) & 197 & (79) & 150 & (49) & 156 & (49) & 177 & (59) & $148 \quad(31)$ & NS \\
\hline PCWP & P mmHg & 21 & $(2.8)$ & 22 & (3) & 25 & (4) & 24 & (4) & 23 & (3) & (3) & NS \\
\hline PASP & $\mathrm{mmHg}$ & 45 & (6) & 46 & (8) & 47 & (7) & 47 & (7) & 47 & (7) & (6) & NS \\
\hline PADP & $\mathrm{mmHg}$ & 21 & (4) & 24 & (5) & 24 & (5) & 24 & (5) & 25 & (4) & (3) & NS \\
\hline RAP & $\mathrm{mmHg}$ & 8.9 & (1.2) & 9.7 & $(1.5)^{\dagger}$ & 10.9 & (1.4) & 10.1 & (1.7) & 11.6 & (1.5) & (1.4) & NS \\
\hline
\end{tabular}

Data is expressed as mean (SEM). Data for 1.5 and $2.5 \mathrm{~h}$ has been omitted for brevity. CI: cardiac index; SVI: stroke volume index; SBP: systolic blood pressure; DBP: diastolic blood pressure; HR: heart rate; SVR: systemic vascular resistance; PVR: pulmonary vascular resistance; PCWP: pulmonary capillary wedge pressure; PASP: pulmonary artery systolic pressure; PADP: pulmonary artery diastolic pressure; RAP: right atrial pressure; NCPAP: nasal continuous positive airway pressure; ANOVA: analysis of variance for repeated measures across all time periods; SNK: Student Newman-Keuls; Ns: nonsignificant. *: p<0.05; **: p<0.01 for SNK test comparing each time period with baseline. ${ }^{\dagger}$ : data for six patients (baseline SVR = 1,272 (128) and RAP = 8.8 $(1.5)$ for these patients). 


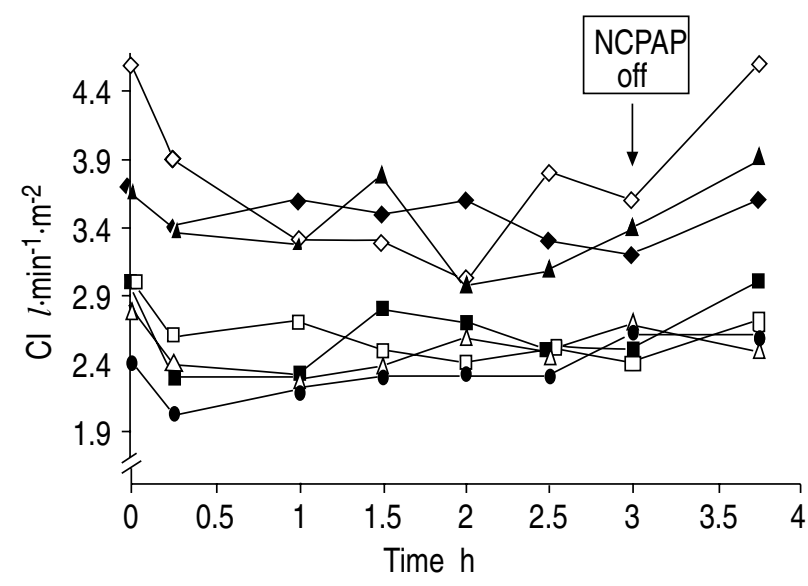

Fig. 1. - Cardiac index (CI) measurements for individual subjects from baseline (time 0) to $45 \mathrm{~min}$ after nasal continuous positive airway pressure (NCPAP) was stopped $(3.75 \mathrm{~h})$. NCPAP was discontinued immediately after recordings were taken at $3 \mathrm{~h}$. Note that the vertical axis is cut off from zero.

to an increase in HR (from 90 to 111 beats. $\mathrm{min}^{-1}$ ) with a lower SV $(41 \mathrm{ml})$ than at baseline $(46 \mathrm{ml})$. His HR fell and SV increased after cessation of NCPAP.

The change in CI was closely matched by a significant fall in SVI $(\mathrm{p}=0.03)$, which was maximal at $2 \mathrm{~h}$, and no significant differences were found between readings at baseline and after withdrawal of NCPAP. Systolic BP $(p=0.0006)$ also rose during NCPAP, as did SVR $(\mathrm{p}=0.003)$. Increases for both were maximal at $2 \mathrm{~h}$. No significant changes were observed in PCWP, PASP, PADP, RAP or $\% \mathrm{SaO}_{2}$ during NCPAP.

On stepwise multiple linear regression analysis, the only significant correlation found was a negative one between baseline SVR and \% fall in CI from baseline at $2 \mathrm{~h}$ (multiple $\mathrm{r}^{2}=0.8 ; \mathrm{p}=0.02$ ). The correlation between the two variables is shown in figure 2 .

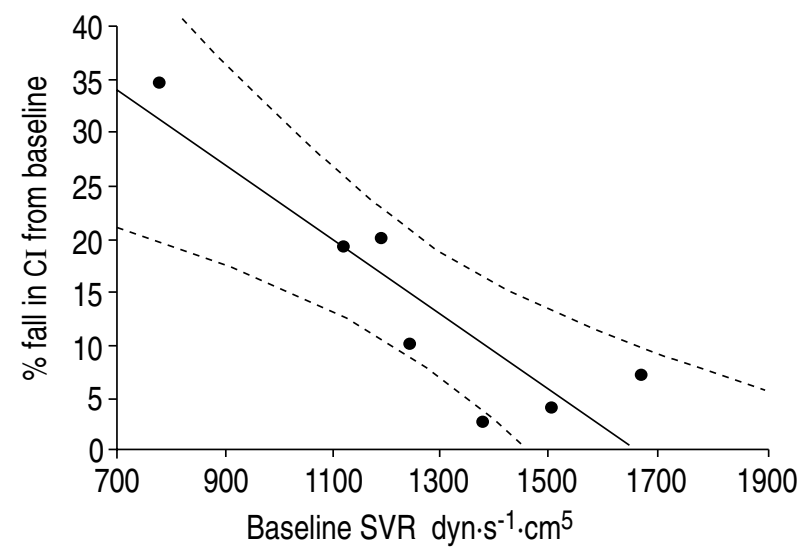

Fig. 2. - The relationship between baseline systemic vascular resistance (SVR) and cardiac index (CI) at $2 \mathrm{~h}$ (expressed as \% fall from baseline). Data points are shown with $95 \%$ confidence limits; $r=-0.89$ and $\mathrm{p}=0.007$ (Pearson product moment correlation coefficient).

\section{Discussion}

The present study demonstrates a significant fall in cardiac output with NCPAP among awake patients with severe stable CHF. These findings differ from those of BRADLEY et al. [15], who studied the awake effects of NCPAP in stable CHF over a shorter time period (10 min). The present study used similar measurement techniques, and our patients resembled the responder group in the other study [15] in terms of PCWP, baseline CI, and ongoing cardiac therapy.

However, atrial fibrillation was seen in 6 out of the 7 patients in the present study, whereas all patients in the other study [15] were in SR, although the change in heart rate during NCPAP was similar in both studies. Our patients also had lower mean LVEFs (23 vs 30.8\%) and lower mean SVRs $\left(1,268\right.$ vs $\left.1,717 \mathrm{dyn} \cdot \mathrm{s}^{-1} \cdot \mathrm{cm}^{5}\right)$ than in the responder group in the other report [15].

The findings of the present study are supported by the observations of PINSKY et al. [22], who reported a fall in CI with positive airway pressure among patients after coronary artery bypass; and also DAVIES et al. [14], who found no improvement in cardiac function or patient performance after 2 weeks of domiciliary NCPAP treatment.

The present study recorded haemodynamic changes in patients with chronic stable $\mathrm{CHF}$, and the findings do not necessarily disagree with reported benefits of positive pressure in patients with acute cardiac failure and pulmonary oedema [7-10]. End-points in these other studies included improvements in arterial blood gases, work of breathing, and requirement for assisted ventilation $[8,9]$. Patients were on different medications, including intravenous inotropic support. Patients in the present study were stable, with no evidence of acute cardiac ischaemia or respiratory failure.

The methodology used in the present study, which is standard for assessing CI and cardiovascular pressures [7, 10, 11, 15], does not permit a precise definition of how NCPAP may lead to a reduction in CI. Positive airway pressure is known to reduce systemic venous return [23]. It also increases functional residual capacity (FRC), thus, elevating both pulmonary vascular resistance and right ventricular (RV) afterload. An increased $\mathrm{RV}$ afterload will reduce pulmonary venous return to the left heart [24, 25], and may also increase RV volume, which in turn can induce leftward shift of the interventricular septum and reduction in left ventricular (LV) volume [24]. Such changes in LV shape and size can adversely affect LV compliance [26].

All of the above mechanisms tend to reduce LV enddiastolic volume and, thus, preload. When contractility is normal, a reduction in preload decreases LV ejection by the Frank-Starling mechanism. As a result, the normal heart is considered to be preload-dependent $[5,6$, $27]$, and normal subjects usually [15, 21], but not always [28], experience a consistent fall in $\mathrm{CO}$ with NCPAP. In contrast, $\mathrm{CO}$ in the failing heart is thought to be more responsive to changes in afterload, as LV contractility is reduced and is relatively unresponsive to changes in diastolic volume $[5,6]$. It has been 
hypothesized, therefore, that the negative effects of a NCPAP induced reduction in preload would be less marked than in the normal heart, and would tend to be outweighed by the beneficial effects of a reduction in $\mathrm{LV}$ afterload, and result in an increase in $\mathrm{LV}$ ejection $[5,6]$.

In the present study, patients with the lowest baseline SVRs had the greatest falls in CI (fig. 2), and were likely to have been significantly affected by the fall in preload. Their baseline SVR, which reflects afterload, was within the normal range, and any benefit derived from a reduction in afterload [4] is likely to have been minimal compared to the reduction in preload. The balance of effects would favour a fall in CI. Furthermore, these patients had the greatest increase in SVR at $2 \mathrm{~h}$ (34-64\%), possibly as a vasomotor response to the fall in CI. In contrast, patients with high baseline SVRs, and thus high afterload, had much smaller reductions in CI. Their cardiac contractility may have been poorer, and, thus, less affected by the fall in preload. They may have gained some benefit from a fall in LV transmural pressure, and this could have counteracted the effect of reduced preload, minimizing the fall in CI. The increase in SVR at $2 \mathrm{~h}$ found in these patients was much lower (less than 20\%).

The possibility that NCPAP reduces the work of breathing, and thus oxygen demand, is worthy of consideration, as a reduction in CI may, therefore, reflect reduced perfusion requirements. This is unlikely to have occurred in the present study, since none of the patients had significant pulmonary disease and all had good $\mathrm{PaO}_{2}$ levels $(>10.5 \mathrm{kPa})$ at rest (table 1). No significant change in $\% \mathrm{SaO}_{2}$ was seen in any patient. Furthermore, the findings of reduced CI and increased SVR are both considered to adversely affect survival in patients with $\mathrm{CHF}$ [29], and would probably have detrimental effects if maintained over a prolonged period of time in our patients.

We did not measure LV transmural pressure, and therefore, the values obtained for RAP, PAPs, and PCWP do not take into account any changes in transmural pressure with NCPAP. However, even if the full mask pressure was transmitted to the intrathoracic space and was subtracted from the observed readings, no significant changes in these pressures were found during NCPAP.

We chose to study patients while awake, since we felt that beneficial respiratory effects of NCPAP during sleep, related to the relief of sleep-disordered breathing and/or hypoxaemia, could have significantly modified the direct cardiac responses to NCPAP. Although three of our patients had sleep study evidence of mild sleep apnoea (table 1), this finding should not influence our awake measurements. None of our patients demonstrated any evidence of Cheyne-Stokes respiration or oxygen desaturation during the study period. Thus, the observed fall in cardiac output is most likely to have been a direct effect of NCPAP.

A number of possible confounding methodological factors in the present study, such as emotional distress, body position, and the effects of concurrent cardiac medication, can be discounted. Patient comfort was a particular concern at all stages, and each subject was well acclimatized to the NCPAP device before haemodynamic studies. Haemodynamic studies were performed in a quiet room with investigators familiar to the subject present throughout the study.

The supine position is very unlikely to have influenced our results, since CI returned to baseline values when NCPAP was discontinued, without a change in posture (table 3). It has been proposed that NCPAP exerts a beneficial effect on CI through a reduction in LV afterload; and it could, therefore, be argued that any benefit would be masked by concurrent therapy with afterload reducing agents. This is unlikely, however, as patients in any of the studies that reported beneficial effects with NCPAP, have also been on such therapy [12, 13, 15]. Furthermore, any therapeutic role for NCPAP in patients with CHF would probably be in addition to existing medication, rather than as a replacement.

Data from the present study do not support the view that low levels of NCPAP can be applied with relative safety as a trial therapy in all patients with poorly controlled CHF [15], although we recognize from previous reports [7-15] that specific subgroups may benefit from NCPAP. Factors that influence an individual patient's response to NCPAP need to be defined before NCPAP can be used in the clinical setting. One possible factor predicting an adverse response to NCPAP is the finding of atrial fibrillation, in view of its presence among most of the patients in the present study.

However, the findings of the present study do not preclude a beneficial effect on cardiac output of NCPAP during sleep in patients with $\mathrm{CHF}$ and a coexisting respiratory sleep disorder, such as sleep apnoea or CheyneStokes respiration, as has previously been reported by MALONE et al. [13]. However, our data suggest that such benefit is likely to be indirect, and related to relief by NCPAP of the adverse cardiac effects from the repetitive Müller manoeuvres and hypoxaemia associated with obstructive apnoeas [13, 30].

We conclude that domiciliary nocturnal NCPAP should not be prescribed as a form of therapy for CHF without first determining the individual patient's haemodynamic response. Further investigation is required to identify factors that might predict cardiac response to NCPAP in such patients.

\section{References}

1. Sullivan CE, Berthon-Jones M, Issa FG, Eves L. Reversal of obstructive sleep apnoea by continuous positive airway pressure applied through the nares. Lancet 1981; i: $862-865$.

2. Sanders MH, Moore SE, Eveslage J. CPAP via nasal mask: a treatment for obstructive sleep apnea. Chest 1983; 83: 144-145.

3. Issa FG, Sullivan CE. Reversal of central sleep apnoea using nasal CPAP. Chest 1986; 90: 165-171.

4. Buda AJ, Pinsky MR, Ingels NB, Daughters GT, Stinson $\mathrm{EB}$, Alderman EL. Effect of intrathoracic pressure on left venticular performance. N Engl J Med 1979; 301: $453-459$. 
5. Pinsky MR, Summer WR, Wise RA, Permutt S, Bomberger-Barnea B. Augmentation of cardiac function by elevation of intrathoracic pressure. J Appl Physiol: Respirat Environ Exercise Physiol 1983; 54: 950-955.

6. Pinsky MR, Matuschak GM, Klain M. Determinants of cardiac augmentation by elevations in intrathoracic pressure. J Appl Physiol 1985; 58: 1189-1198.

7. Grace MP, Greenbaum DM. Cardiac performance in response to PEEP in patients with cardiac dysfunction. Crit Care Med 1982; 10: 358-360.

8. Räsänen J, Heikkilä J, Downs J, Nikki P, Väisänen I, Vitanen A. Continuous positive airway pressure by facemask in acute cardiogenic pulmonary edema. Am J Cardiol 1985; 55: 296-300.

9. Bersten AD, Holt AW, Vedig AE, Skowronski GA, Baggoley CJ. Treatment of severe cardiogenic pulmonary edema with continuous positive airway pressure delivered by face mask. N Engl J Med 1991; 325: 18251830.

10. Baratz DM, Westbrook PR, Shah PK, Mohsenifar Z. Effect of nasal continuous positive airway pressure on cardiac output and oxygen delivery in patients with congestive heart failure. Chest 1992; 102: 1397-1401.

11. Pinsky MR, Marquez J, Martin D, Klain M. Ventricular assist by cardiac-cycle specific increases in intrathoracic pressure. Chest 1987; 91: 709-715.

12. Takasaki Y, Orr D, Popkin J, Rutherford R, Liu P, Bradley TD. Effect of nasal continuous positive airway pressure on sleep apnea in congestive heart failure. Am Rev Respir Dis 1989; 140: 1578-1584.

13. Malone S, Liu PP, Holloway R, Rutherford R, Xie A, Bradley TD. Obstructive sleep apnoea in patients with dilated cardiomyopathy: effects of continuous positive airway pressure. Lancet 1991; ii: 1480-1484.

14. Davies RJO, Harrington KJ, Ormerod OJM, Stradling JR. Nasal continuous positive airway pressure in chronic heart failure with sleep-disordered breathing. Am Rev Respir Dis 1993; 147: 630-634.

15. Bradley TD, Holloway RM, McLaughlin PR, Ross BL, Walters J, Liu PP. Cardiac output response to continuous positive airway pressure in congestive heart failure. Am Rev Respir Dis 1992: 145: 377-382.

16. Criteria Committee, New York Heart Association, Inc. In: Diseases of the Heart and Blood Vessels. Nomenclature and Criteria for Diagnosis. 6th edn. Boston, Little, Brown and Co., 1964; p. 114

17. Rechtschaffen A, Kales A. In: A Manual of Standardized Terminology, Techniques and Scoring System for Sleep Stages in Human Subjects. Washington, D.C., National Institutes of Health, 1968; Publication 204.

18. Gould GA, Whyte KF, Rhind GB, et al. The sleep hypopnea syndrome. Am Rev Respir Dis 1988; 137: 895-898.

19. Forrester JS, Ganz W, Diamond G, McHugh T, Chonette DW, Swan HJC. Thermodilution cardiac output determination with a single flow-directed cath-eter. Am Heart J 1972; 83: 306-311.

20. Grossman W, McLaurin LP. Clinical measurement of vascular resistance and assessment of vasodilator drugs. In: Grossman W, ed. Cardiac Catheterisation and Angiography. Philadelphia, Lea \& Febiger, 1980; pp. 116-123.

21. Montner PK, Greene ER, Murata GH, Stark DM, Timms M, Chick TW. Hemodynamic effects of nasal and face mask continuous positive airway pressure. Am J Respir Crit Care Med 1994; 149: 1614-1618.

22. Pinsky MR, Desmet J-M, Vincent JL. Effect of positive end-expiratory pressure on right venticular function in humans. Am Rev Respir Dis 1992; 146: 681-687.

23. Jardin F, Farcot J-C, Guéret P, Prost J-F, Ozier Y, Bourdarias J-P. Echocardiographic evaluation of ventricles during continuous positive airway pressure breathing. J Appl Physiol: Respirat Environ Exercise Physiol 1984; 56: 619-627.

24. Jardin F, Farcot J-C, Boisante L, Curien N, Margairaz A, Bourdarias J-P. Influence of positive end-expiratory pressure on left ventricular performance. $N$ Engl J Med 1981; 304: 387-392.

25. Jardin F, Delorme G, Hardy A, Auvert B, Beauchet A, Bourdarias J-P. Re-evaluation of hemodynamic consequences of positive pressure ventilation: emphasis on cyclic right ventricular afterloading by mechanical lung inflation. Anesthesiology 1990; 72: 966-970.

26. Haynes JB, Carson SD, Whitney WP, Zerbe GO, Hyers TM, Steele P. Positive end-expiratory pressure shifts left ventricular diastolic pressure-area curves. $J \mathrm{Appl}$ Physiol: Respirat Environ Exercise Physiol 1980; 48: 670-676.

27. Fewell JE, Abendschein DR, Carlson CJ, Murray JF, Rapaport E. Continuous positive-pressure ventilation decreases right and left ventricular end diastolic volumes in the dog. Circ Res 1980; 46: 125-132.

28. Leech JA, Ascah KJ. Hemodynamic effects of nasal CPAP examined by Doppler echocardiography. Chest 1991; 99: 323-326.

29. Braunwald E, Grossman W. Clinical aspects of heart failure. In: Braunwald E, ed. Heart Disease: A Textbook of Cardiovascular Medicine. 4th edn. Philadelphia, W.B. Saunders, 1992; pp. 444-463.

30. Tolle FA, Judy WV, Yu P-L, Markand ON. Reduced stroke volume related to pleural pressure in obstructive sleep apnoea. J Appl Physiol: Respirat Environ Exercise Physiol 1983; 55: 1718-1724. 